\title{
Conocimiento previo y resumen de un texto argumentativo
}

\section{Prior knowledge and outline of an argumentative text}

María Nélida Pérez López ${ }^{1}$

\section{Resumen}

En este artículo se reporta una investigación que tuvo como objetivo determinar la correlación entre el conocimiento previo y la comprensión lectora de un texto argumentativo en estudiantes que iniciaron sus estudios universitarios. Se determinó el nivel de conocimiento relativo a los dos tópicos tratados en un artículo de opinión de lectura posterior, así como el nivel de adecuación y precisión de las macroproposiciones (tesis, argumentos y conclusión) del esquema y del resumen elaborados a partir de dicho artículo. Los resultados indican que no hubo correlación entre la identificación adecuada y precisa de las ideas y el nivel de conocimiento. Finalmente, se analiza la incidencia de algunos factores, como el referente al modelo de contexto desde el que los estudiantes asumieron el proceso de comprensión.

\section{Palabras clave:}

lectura, resumen, conocimiento previo, texto argumentativo.

\section{Abstract}

This article gives an account of an investigation that was geared towards determining the correlation between previous knowledge and the reading comprehension of an argumentative text in students who are beginning their college studies. Both determined the level of relative knowledge of two topics in a posterior reading of an article, as well as the level of adjustment and precision to the macropropositions (thesis, arguments and conclusion) of the scheme and the summary elaborated from the article. The results indicate that there was no correlation between the suitable and precise identification of the ideas and the level of knowledge. Finally, the incidence of some factors is analyzed, for example the reference to the context model from which the students assumed the understanding process.

\section{Keywords:}

reading, summary, prior knowledge, argumentative text.

Artículo recibido el 22 de abril de 2012 y aprobado el 8 de febrero de 2013

1 Universidad Simón Bolívar, Caracas, Venezuela. Correo electrónico: mnperez@usb.ve 
En esta investigación iniciamos un estudio de la incidencia del conocimiento previo sobre el proceso de comprensión de un texto argumentativo, cuya lectura no tiene por objeto adquirir información con la finalidad de incrementar su dominio; en este proceso el lector utiliza su conocimiento con un doble propósito: comprender el texto, lo que incluye comprenderlo como la expresión de una opinión (van Dijk, 1999), y evaluar tal opinión -el razonamiento que la sustenta- para determinar hasta qué punto podría o no suscribirla. La habilidad para resumir, además de un indicador de comprensión, es clave en el desarrollo de competencias relativas al uso de estrategias de lectura crítica en el ámbito educativo, donde ha tendido a establecerse una vinculación entre resumen y conocimiento previo: "los conocimientos previos son necesarios para formar la macroestructura y, a la vez, los procesos que dirigen la formación de la macroestructura dirigen también la búsqueda de conocimientos previos relevantes" (Sánchez, 1993, p. 101).

El estudio del resumen ha estado relacionado, principalmente, con su función didáctica, como estrategia de estudio y, por tanto, se ha centrado en el texto expositivo, cuya lectura se hace con la finalidad de aprender. Ahora bien, ¿el conocimiento previo tendrá tal influencia sobre un texto argumentativo, cuya lectura, insistimos, se hace con otra intención? Si ello resultare así, este conocimiento podría tener una primera incidencia sobre la selección de las ideas relevantes del texto leído y, luego, durante el análisis, una segunda, pues será necesario para determinar la intención del autor, las creencias que sustentan su acto persuasivo y las estrategias que emplea, así como para evaluar la suficiencia y la pertinencia de los argumentos utilizados, lo que llevará al lector a apoyarlo o rebatirlo, según se sea o no convencido de su posición por el escritor. En este trabajo, solo reportaremos resultados de esa primera incidencia, comprensiva; sin embargo, dejaremos abiertas algunas puertas durante la fase de análisis de los resultados, específicamente de los referidos a la elaboración de la estructura semántica del esquema de ideas y del resumen con miras a su tratamiento posterior, en virtud de otros propósitos de investigación y otros procesos de lecto-escritura.

\section{La lectura y el resumen}

El resumen es un texto de amplio uso en el ámbito académico. En múltiples situaciones, se constituye en el paso previo de la construcción de muchos otros (revisiones teóricas, informes, comentarios, reseñas, etc.). Su elaboración ha sido establecida como una de las características fundamentales de un buen lector, quien, al hacerlo, utiliza un conjunto de operaciones mentales, definidas por Kintsch y van Dijk (1978) como macrorreglas de supresión, generalización y construcción, con la finalidad de elaborar su macroestructura, al tiempo que atiende a la superestructura del texto -lo que supone tener en cuenta la organización de las ideas en función de las relaciones que se establecen entre ellas-, $y$ elabora un modelo mental tanto de aquello de lo que el texto trata como de la situación en la que su lectura se inscribe. Por ende, lo que generalmente se recuerda del texto es tal modelo mental (van Dijk, 1999, 2000).

Y debido a que la lectura es un proceso de interrelación entre lector y texto, en él ha de intervenir el conocimiento con el que el lector se acerca al texto: "El resumen exige la identificación de las ideas principales y de las relaciones que entre ellas establece el lector de acuerdo con sus objetivos de lectura y conocimientos previos a ella" (Solé, 2006, p. 131). De ahí que en la investigación que se ha desarrollado a lo largo de los últimos años sobre el resumen, el conocimiento haya ido adquiriendo cada vez mayor importancia a causa de la aparición de nuevos enfoques que resaltan el papel activo del lector en el proceso de comprensión. Ello explica por qué, por ejemplo, Kahmi-Stein (1997), utilizó textos de conocimiento general de tal forma que éste no interfiriera sobre la evaluación de las habilidades para redactar resúmenes, y, en cambio, Zipitria, Elorriaga y Arruarte (2007) propusieron la evaluación de los conocimientos (nivel del aprendiz, conocimientos previos sobre el tema y contexto educativo), como uno de los parámetros que han de 
ser tenidos en cuenta en la evaluación automatizada de esta habilidad.

La competencia lectora, además, ha estado ligada a un mayor control en la aplicación de las estrategias y a la conciencia de la organización del texto, lo que puede deberse a que la comprensión esté controlada por una superestructura específica a través de la que los individuos relacionan información nueva con información conocida, ya sea del texto, del contexto o de su sistema de conocimiento general (Kintsch y van Dijk, 1978). Por tanto, no sería lo mismo resumir un texto expositivo cuyo autor tiene un propósito principalmente informativo en relación con el lector, que otro argumentativo cuyo propósito persuasivo genera una dinámica dialógica de mayor intensidad, pues, como indica Alonso Tapias (2005, p. 78), "comprenderlos en profundidad implica ser capaces de identificar y valorar la intención con que han sido escritos”. Aún más, en opinión de González Nieto (2001, p. 288), "cada tipo de texto presenta sus propios problemas. Es más fácil ser 'objetivo' y fiel al original al resumir un texto informativo que un texto de opinión".

En consecuencia, la lectura se constituye en un complejo proceso, durante el cual se elaboran representaciones mentales jerárquicas de las ideas, que no son simples copias del texto, sino "el resultado de procesos estratégicos de construcción de sentido que pueden utilizar elementos del texto, de lo que los usuarios del lenguaje saben acerca del contexto y elementos de las creencias que estos usuarios ya tenían antes de iniciar la comunicación" (van Dijk, 2000, p. 43). Las creencias son definidas por van Dijk como unidades o representaciones mentales involucradas en el procesamiento de la información y, por ende, del discurso. El mundo se comprende y se interpreta a través del sistema de creencias que tienen los individuos; "las creencias constituyen el mundo-según-nosotros" (van Dijk, 1999, p. 43) y pueden ser fácticas o evaluativas. Las primeras incluyen el conocimiento que se comparte socialmente o que ha sido aceptado sobre la base de criterios socialmente establecidos; las segundas, las opiniones, juicios o puntos de vista que se posee sobre la gente que tiene determinadas creencias o sobre las rela- ciones que esa gente tiene con el mundo (van Dijk, 1999, 2005). Y si bien ambos tipos de creencias son especialmente relevantes en la construcción de la representación mental de un texto, en esta primera parte de la investigación nos ocuparemos, básicamente, del conocimiento previo, en tanto creencia fáctica -lo que se sabe de algo- porque nuestro objetivo es determinar su incidencia sobre la elaboración de un resumen. Sin embargo, esto no nos lleva a descartar toda consideración de las posibles opiniones -lo que se piensa de algo-, elemento clave en el procesamiento de un texto argumentativo, pues "muchas de las presuposiciones implícitas de la argumentación se derivan de un conocimiento general de grupo que está fundado ideológicamente o que proviene de modelos de sucesos mentales personales también de base ideológica" (van Dijk, 2005, p. 314).

\section{El conocimiento}

En líneas generales, y como ya hemos indicado, la investigación que se ha hecho sobre el conocimiento previo ha tendido a estudiar y valorar su peso en la comprensión de textos especializados cuya lectura tiene una finalidad epistémica. En el área de las ciencias y específicamente de su didáctica, por ejemplo, los trabajos de Solaz-Portolés y Sanjosé $(2007,2008)$ reportan la importancia de la base de conocimientos -especialmente declarativos- así como de su organización estructural -la que permite integrar con éxito el conocimiento nuevo- en la resolución de problemas, por lo que cuando el conocimiento previo es bajo, recomiendan ofrecer al aprendiz textos de una alta coherencia vinculados con los modelos mentales que posea. A pesar de ello, afirman que, en problemas para cuya resolución no se requiere de la elaboración de modelos mentales sino simplemente de una representación mental proposicional, el conocimiento previo ofrece una escasa influencia; y que este conocimiento solo hace posible discriminar adecuadamente cuándo deben ejecutarse pocos modelos mentales para la resolución del problema. 
McNamara (2004), quien comenzó estudiando los efectos del conocimiento previo sobre la cohesión textual, ha terminado por establecer que su uso es una estrategia lectora más cuya aplicación, junto con otras (lectura previa, predicción, inferencia, resumen y autoexplicación), ayuda al individuo a desarrollar su comprensión, debido a la evidencia del primerísimo papel que las habilidades lectoras, $y$ específicamente las inferencias basadas en el texto, la lógica y el conocimiento, han demostrado tener en la comprensión de textos de menor o mayor cohesión.

Asimismo, Castejón et al. (2004) han concluido que entre la organización conceptual del conocimiento, la habilidad intelectual general y la adquisición del conocimiento existe un efecto aditivo en lugar de compensatorio. Si bien altos niveles de organización conceptual y de habilidad intelectual contribuyen a que el sujeto alcance altos niveles de conocimiento, un alto nivel de organización conceptual no compensa una baja habilidad intelectual en la adquisición de nuevo conocimiento.

Trabajos anteriores ya habían demostrado que el pobre desempeño de los lectores reside principalmente en la dificultad de generar inferencias porque confían prioritaria o excesivamente en su conocimiento previo (McCormick, 1992; McGinitie, Maria y Kimmel, 1986; Wine, Graham y Prock, 1993), porque no pueden crear modelos mentales acordes con el texto que les permitan determinar la información relevante (Bernardo y Stenberg, 1991; Gray et al., 1993) o porque las restricciones de la memoria de trabajo limitan la habilidad de los lectores menos hábiles que intentan comprometerse en un procesamiento inferencial (Long, Oppy y Seely, 1994).

Algunos enfoques instructivos han planteado el estudio del conocimiento a partir de propuestas teóricas que centran su acción en el sistema cognitivo del individuo. En ellas, el modelo de redescripción representacional de Karmiloff-Smith (1994) ha tenido gran importancia. Desde esta perspectiva, las demandas de cada contexto exigirían la construcción de modelos mentales en los que el conocimiento se redescribiría -se reorganizaría- en función de un nuevo marco de relaciones conceptuales; en el ámbito de la didáctica de la ciencia al que hemos hecho alusión, por ejemplo, el conocimiento popular, intuitivo, implícito, no sería sustituido por el científico, sino redescrito, explicado, en modelos de mayor complejidad y explicitado a través de variados sistemas de representación (v.g., Pozo, 2002); lo que pareciera un paso hacia la necesidad de atender a las dimensiones social y cultural del conocimiento.

Una propuesta en la que se integran con el cognitivo, los enfoques social y discursivo, es la que presenta van Dijk (2002), para quien todavía falta mucho por hacer en este campo: "Si bien estamos de acuerdo en que la mayor parte del aprendizaje se realiza a través del procesamiento del discurso, hay muchos aspectos en los procesos de adquisición del conocimiento que aún no se conocen" (p. 44), debido a que ninguna de las disciplinas que hasta ahora lo han estudiado ha podido ofrecer una visión que explique la interfaz entre discurso y conocimiento, y más específicamente, de cómo se hace presente en el texto y en el habla (van Dijk, 2010). De allí que proponga abordarlo desde una teoría multidisciplinaria que comience por definir qué se entiende por conocimiento, dé cuenta de los tipos de conocimiento, de las estructuras en las que se representa mentalmente, de cómo son utilizadas en el procesamiento del discurso, de cómo se adquieren los distintos tipos de conocimiento nuevo y de cómo se integran en el sistema que ya se posee, $y$, finalmente, explique los factores sociales, culturales y políticos que intervienen en su reproducción en los distintos grupos y culturas.

Para establecer una tipología, van Dijk (2002) considera dos factores: la persona que adquiere o comparte el conocimiento (personal, grupal o cultural) y los objetos de conocimiento (de eventos específicos o de las propiedades de los eventos generales; de eventos históricos o de sus estructuras sociales o políticas). El conocimiento personal es individual y desconocido para el receptor hasta que el individuo no lo verbalice, de manera que no pueden hacerse presuposiciones acerca de él, a diferencia del conocimiento grupal que, evidentemente, 
es compartido por los miembros de un grupo, fuera de los cuales es desconocido. El conocimiento cultural, o de terreno común, es compartido por los miembros de una cultura y adquirido a través del hogar, la escuela y los medios de comunicación. Es la "base de toda cognición social" (van Dijk, 2002, p. 49), pues los conocimientos personal y grupal se asientan sobre el cultural.

El conocimiento se representa a través de modelos mentales, esto es, del "modo en que las personas construyen, de manera subjetiva, los eventos del mundo a partir de sus experiencias" (van Dijk, 2002, p. 50). Si los hechos se generalizan, se construyen modelos mentales generales y abstractos que son compartidos socialmente y aplicados en muchas situaciones por muchas personas, porque en la comprensión y la producción del discurso, ellas activan, seleccionan y expresan el conocimiento en virtud de sus modelos de contexto, esto es, de las representaciones mentales que han creado sobre las propiedades que son pertinentes al hecho comunicativo en el que están inmersas. La ubicación (lugar y tiempo), las circunstancias y, especialmente, los roles (participante, profesional, social) definen la perspectiva desde la que se asume un modelo de contexto. Sin embargo, esto no significa que el conocimiento sea el mismo para toda la comunidad, pues la diversidad de las experiencias personales y, consecuentemente, de los modelos mentales, genera conocimientos diferentes, que pueden o no ser compartidos por los miembros de una comunidad. Lo común, según esta propuesta teórica que apunta a una organización jerárquica, es el conocimiento básico, prototípico, superficial. Asimismo, las propiedades del acto comunicativo determinan la activación de un conocimiento que puede ser el mínimamente requerido para elaborar un modelo mental coherente o altamente detallado y lleno de asociaciones con otros conocimientos, porque son los modelos de contexto los que explican cómo el conocimiento personal que se tiene sobre las acciones o los hechos representados en los modelos de acontecimiento han de ser expresados o comprendidos en el discurso.

\section{Metodología}

\section{Objetivo y problema}

El propósito de esta investigación fue determinar la incidencia del conocimiento previo del estudiante sobre la elaboración de la síntesis de un texto argumentativo (artículo de opinión), en dos formatos, esquema de ideas y resumen, en atención al problema de estudio que nos planteamos: ¿qué tipo de correlación se establece entre el nivel de competencia del sujeto en la elaboración de la macroestructura de un texto argumentativo y, el conocimiento cultural, específicamente el relativo a su temática, activado y comunicado por el sujeto previamente a su lectura?

\section{Muestra}

La muestra de este estudio estuvo constituida por 44 estudiantes de la asignatura Lenguaje I (primer trimestre de primer año) de la Universidad Simón Bolívar, con una edad promedio de 17,3 años. Fueron identificados como E1, E2...E44 a partir del orden de entrega del primer test. Los alumnos, de diferentes especialidades de Ingeniería, Arquitectura o Ciencias Básicas, al ingresar, deben cursar un ciclo básico de un año de duración (en un régimen trimestral), con materias comunes (Lenguaje, Ciencias Sociales, Idiomas, Matemática y Física).

\section{Instrumentos y procedimiento de recolección y análisis del corpus}

En las primeras semanas del trimestre se les solicitó, como evaluación de diagnóstico, dar respuesta a dos test, cada uno de ellos de una hora de duración. En la primera clase se aplicó el test 1, de respuesta abierta, el cual consistió en una prueba de conocimiento sobre dos aspectos temáticos que se interrelacionaban en el desarrollo del artículo de opinión que posteriormente leerían: fin del mundo y cambio climático; tópicos de amplia difusión en la actualidad (no solo adquiridos por el individuo en su educación formal, sino también a través de los medios de comunicación social) y en total interrelación por ser, el último, posible causa del primero. Estos temas forman parte, por tanto, del 
conocimiento cultural (compartido por la comunidad) que se sustenta sobre criterios establecidos por estudios científicos, pues independientemente de que los sujetos crean o no que el cambio climático será causa del fin del mundo (opinión), saben -con mayor o menor competencia- lo que es el cambio climático, sus causas y consecuencias, así como las versiones que sobre el fin del mundo ha habido a lo largo de la historia de su cultura (conocimiento).

En este sentido, les pedimos elaborar dos textos expositivos donde expresaran la información de que dispusiesen en cuanto a cambio climático (causas, consecuencias generales y particulares relacionadas con países desarrollados, no desarrollados o en vías de desarrollo y su ubicación geográfica) y fin del mundo (versiones religiosa, de ciencia ficción, histórica y científica).

Este test permitió determinar el conocimiento personal antes de dar respuesta al test 2 (esquema y resumen), lo que en ningún caso puede hacer presuponer que el conocimiento expresado abarca la totalidad del conocimiento poseído por cada individuo en relación con los temas dados; tan solo hemos intentado hacer explícito, verbal, un conocimiento personal al que, como ya hemos expresado, únicamente se puede acceder una vez sea comunicado por el individuo, que fue, en este caso, quien decidió cuál y cuánto de su conocimiento poner a nuestra disposición. Del mismo modo, hemos tenido en cuenta que "no todo el conocimiento puede ser siempre accesible activamente" (van Dijk, 1999, p. 59).

En la clase siguiente, aplicamos el segundo test, donde solicitamos la redacción de un esquema de ideas y de un resumen de un texto argumentativo, específicamente Noticias del fin del mundo, del escritor español Antonio Muñoz Molina.

La macroestructura (tesis, argumentos y conclusión) fue identificada y jerarquizada por un grupo de cinco expertos, profesores de Lenguaje de la misma universidad. La elaboración de la macroestructura requería la aplicación de las macrorreglas ampliamente conocidas: supresión-selección, generalización y construcción (Kinstch y van Dijk, 1978). Así, la tesis debía ser inferida tomando en cuenta la intención discursiva (argumentativa) del autor y algunos elementos claves del texto (como el título, la estructura argumentativa y la pesimista conclusión). El primer argumento debía ser construido a partir de un conjunto de referencias sobre la llegada tantas veces anunciada del fin del mundo. Para los argumentos 2 y 3 podían suprimir información, con la finalidad de seleccionar algunas proposiciones de las que generalizar las macroproposiciones finales, y la conclusión debía ser seleccionada.

Para registrar y analizar los resultados, establecimos una correspondencia entre el conocimiento previo solicitado y el contenido macroproposicional (MP) de la tesis y los argumentos del texto objeto de lectura, de lo que da cuenta la siguiente tabla:

Tabla 1. Estructura comparativa entre el conocimiento previo requerido y la macroestructura del texto argumentativo

\begin{tabular}{|c|c|}
\hline Conocimiento previo & $\begin{array}{l}\text { Macroestructura del texto argu- } \\
\text { mentativo }\end{array}$ \\
\hline $\begin{array}{l}\text { Fin del mundo: visión } \\
\text { científica (cambio climá- } \\
\text { tico). }\end{array}$ & $\begin{array}{l}\text { Tesis (MP1) } \\
\text { El fin del mundo podría hacerse } \\
\text { realidad a través del cambio } \\
\text { climático. }\end{array}$ \\
\hline $\begin{array}{l}\text { Versiones del fin del } \\
\text { mundo dadas por la } \\
\text { religión, la ciencia ficción } \\
\text { y la historia. }\end{array}$ & $\begin{array}{l}\text { Argumento 1(MP2) } \\
\text { A lo largo de la historia del hom- } \\
\text { bre, el fin del mundo ha sido un } \\
\text { tema de preocupación perma- } \\
\text { nente, que ha generado muchas } \\
\text { versiones (religiosas, de ciencia } \\
\text { ficción, históricas). }\end{array}$ \\
\hline $\begin{array}{l}\text { Consecuencias gene- } \\
\text { rales y particulares } \\
\text { del cambio climático, } \\
\text { relacionadas con países } \\
\text { desarrollados, no desa- } \\
\text { rrollados o en vías de } \\
\text { desarrollo y su ubicación } \\
\text { geográfica. }\end{array}$ & $\begin{array}{l}\text { Argumento } 2 \text { (MP3) } \\
\text { Según el informe de la ONU, en } \\
\text { el plazo de unos pocos años, } \\
\text { podría haber enfermedades, } \\
\text { inundaciones, sequías, ruina } \\
\text { agraria, éxodo humano en busca } \\
\text { de alimentos y agua, etc. } \\
\text { Los más afectados serán los } \\
\text { países más pobres. }\end{array}$ \\
\hline \multirow[t]{2}{*}{$\begin{array}{l}\text { Causas del cambio } \\
\text { climático. }\end{array}$} & $\begin{array}{l}\text { Argumento } 3 \text { (MP4) } \\
\text { Mientras tanto, los gobernantes } \\
\text { están arruinándoles el mundo } \\
\text { a las próximas generaciones, } \\
\text { porque no solo no se ocupan de } \\
\text { solucionar el problema, sino que } \\
\text { lo agudizan con sus acciones } \\
\text { (creación de fronteras, especula- } \\
\text { ción inmobiliaria, despilfarro del } \\
\text { agua, entre otras). }\end{array}$ \\
\hline & $\begin{array}{l}\text { Conclusión (MP5) } \\
\text { "Lo más triste no es pensar en } \\
\text { las cosas que ya no tienen reme- } \\
\text { dio; es saber que la insensatez } \\
\text { humana no va a dejarnos hacer } \\
\text { las que todavía están en nuestra } \\
\text { mano para aliviar el desastre". }\end{array}$ \\
\hline
\end{tabular}


Posteriormente, procedimos a crear un baremo de cuatro niveles que nos permitiera determinar el conocimiento expresado en cada una de las macroproposiciones, así como establecer los niveles de adecuación y precisión semántica de identificación de cada macroproposición y, por tanto, de la macroestructura del esquema y del resumen: $0=$ ausente o nulo; 1 = bajo; 2 = medio; 3 = alto.

Para evaluar la pertinencia de cada macroproposición, manteniendo semejantes indicadores entre ambos formatos, esquema y resumen, partimos principalmente de dos propiedades del resumen que atienden a la comprensión del texto fuente (Núñez y del Teso, 1999): la adecuación al texto, o correspondencia entre su información y la de la macroproposición elaborada, y la precisión, o reproducción del sentido del texto sin tergiversaciones, ya sea por exceso o por defecto. Ello no impide que otras propiedades de mayor relación con la producción del resumen hayan sido consideradas: la textualidad (articulación de las ideas de manera cohesiva y coherente), la autonomía (de qué trata el texto, pero también qué dice al respecto) y la economía y proporcionalidad (información básica, que mantiene el rango de relevancia de las ideas, reducida a lo indispensable en su expresión lingüística).

\section{Presentación y análisis de resultados}

En un sentido general, el promedio del nivel de conocimiento previo exhibido por los alumnos fue de 1,82. El conocimiento explicitado en mayor medida estuvo relacionado con los argumentos $3(\overline{\mathrm{x}}=2,14)$ y $2(\bar{x}=2,09)$, los que se corresponden respectivamente con las causas y las consecuencias del cambio climático (tabla 2). Sin embargo, los datos muestran que no hubo correspondencia entre los niveles de conocimiento previo y los de identificación y elaboración de la idea (tabla 3 ), pues siendo estos los argumentos de mayor promedio de conocimiento son, a su vez, los de menor nivel de elaboración tanto en el esquema ( $A 3: \overline{\mathrm{X}}=0,64 ; \mathrm{A} 2: x=1,52)$ como en el resumen $(A 3: \bar{x}=0,77 ; A 2: \bar{x}=1,43)$. Veamos ahora en detalle cada macroproposición.
Tabla 2. Promedio ( $\bar{x})$ de conocimiento previo por macroproposición (tesis, argumento) y macroestructura

\begin{tabular}{|c|c|c|c|c|}
\hline MP1 (tesis) & MP2 (A1) & MP3 (A2) & MP4 (A3) & ME (global) \\
\hline 1,34 & 1,70 & 2,09 & 2,14 & 1,82 \\
\hline
\end{tabular}

El promedio del conocimiento previo relativo a la tesis, específicamente del cambio climático como teoría científica del fin del mundo, en el test 1 , fue el más bajo $(\overline{\mathrm{x}}=1,34)$; no obstante, a diferencia de lo ocurrido con los argumentos ya indicados, los promedios de su adecuación y precisión en el esquema $(\overline{\mathrm{x}}=1,52)$ y en el resumen $(\overline{\mathrm{x}}=1,48)$ son cercanos.

Tabla 3. Promedio $(\bar{x})$ del nivel de adecuación y precisión de cada macroproposición en el esquema y en el resumen

\begin{tabular}{|c|c|c|c|c|c|}
\hline & MP1 (T) & MP2 (A1) & MP3 (A2) & MP4 (A3) & MP5 (C) \\
\hline Esquema & 1,52 & 1,55 & 1,52 & 0,64 & 1,34 \\
\hline Resumen & 1,48 & 1,68 & 1,43 & 0,77 & 1,25 \\
\hline
\end{tabular}

Entre el conocimiento previo y el nivel de elaboración de las macroproposiciones relativas a la tesis en el esquema, hay una correlación positiva débil ( $\mathrm{r}=0,4288)$, que, en el resumen, se pierde completamente $(r=0,0850)$ (tabla 4). De manera que la posesión de un conocimiento afín con el del texto argumentativo por leer no parece ser un indicador de la habilidad comprensiva para determinar la tesis. No obstante, hemos de advertir que el 93,75\% de los que manifestaron tal conocimiento previamente, en el test de conocimiento (36,36\%), en términos de creencia evaluativa (y no tan sólo fáctica), identificaron esta idea, con mayor o menor nivel de precisión, como tesis o argumento en alguno o en ambos formatos. A pesar de que otros, que no hicieron explícita su opinión o expresaron la contraria, también pudieron inferirla, este aspecto debe ser tenido en cuenta para futuros análisis, pues el poseer una posición crítica coincidente con la del autor del texto podría ser un factor de incidencia en la selección de sus ideas (v. selección de argumento 3 y conclusión). 
Tabla 4. Correlación entre el nivel de conocimiento previo y el nivel de elaboración de cada macroproposición en el esquema y en el resumen

\begin{tabular}{|c|c|c|}
\hline & Esquema & Resumen \\
\hline Tesis & 0,4288 & 0,0850 \\
\hline Argumento 1 & 0,2687 & 0,3044 \\
\hline Argumento 2 & 0,2289 & 0,0661 \\
\hline Argumento 3 & $-0,2198$ & $-0,1879$ \\
\hline
\end{tabular}

Aunque con diferentes niveles de precisión, $45,45 \%$ de los sujetos derivó conscientemente la tesis en el esquema. Otro 20,45\% lo hizo de manera intuitiva, entendiéndola como segundo argumento. El resto, que optó por seleccionar del texto una oración ("No hay peores Apocalipsis que los de la realidad ni profecías más terribles que las del sentido común”), pudo verse afectado por la no aparición expresa de una idea que debía ser inferida en lugar de seleccionada o construida; así ocurrió con el primer argumento, cuya elaboración de nivel 2 o 3 exigió la aplicación de la macrorregla de construcción, que fue empleada por el 52,27\% de los sujetos en el esquema y por el $59,1 \%$ en el resumen. Algunos de ellos $(38,63 \%)$ construyeron esta macroproposición (MP1) interpretándola como tesis quizás porque se vieron influidos por el peso del conjunto de proposiciones relativas a ella, concretado (aparentemente) en su extensión $(42,18 \%$ del total de palabras del texto). En otros, esta acción tal vez pudo estar motivada por un aspecto relacionado con la percepción de la relevancia informativa: dieron mayor peso a las versiones pasadas del fin del mundo que a la posible versión futura: cambio climático. Es también posible que la causa pueda estar en la no consideración de la intención persuasiva del autor $y$, consecuentemente, del tipo de texto (artículo de opinión) y de su superestructura discursiva (argumentativa). Parecería haber en esta concepción un acercamiento al texto argumentativo desde el conocimiento de un texto expositivo que intenta reproducir el conjunto de su información en una idea u oración temática que es explicada a través de derivación de otras ideas (hechos en secuencia).
(E29) (Esquema) Antonio Muñoz plantea las diversas percepciones del fin del mundo y de cómo las ha adoptado la humanidad

(Resumen). En el texto se relata el proceso evolutivo de las creencias por las que se ha inclinado el hombre acerca del fin del mundo, comenzando con las creencias religiosas, hasta llegar a las hipótesis científicas y a la ciencia ficción, ninguno con basamentos fuertes.

A ello puede atribuírsele, por ende, el que casi la mitad de los sujetos que concibieron el argumento 1 como tesis, derivara, en el resumen, lo que consideró el primer argumento como una explicación de la tesis. De allí que la progresión discursiva de estos textos se diera en términos de idea-explicación (en una o varias oraciones), en consistencia con la que entendían era la superestructura y, como advertiremos más adelante, con la asunción de la tesis como argumento 2 .

(E40) (Resumen) El texto "Noticias del Fin del Mundo" escrito por Antonio Muñoz Molina, plantea las diferentes ideas acerca del fin del mundo durante la historia. Cuando las profecías religiosas pierden su credibilidad, la ciencia ficción toma su puesto, ahora el lanzamiento de bombas atómicas o la invasión extraterrestre serán la causa del fin del mundo. Después de la Guerra Fría las imaginaciones apocalípticas se concentrarán con los desastres cósmicos.

Al igual que en la tesis, la correlación que se da entre el nivel del conocimiento previo y el de la adecuación y precisión del argumento 1 en cada formato, esquema y resumen, es débil $(r=0,2687$; $\mathrm{r}=0,3044$, respectivamente). El promedio de conocimiento previo expuesto por los sujetos en relación con el primero de los argumentos fue de 1,70 (tabla 2). Más bajos fueron los promedios de pertinencia del argumento en el esquema $(\overline{\mathrm{x}}=1,55)$ $y$ en el resumen $(\bar{x}=1,68)$ (tabla 3 ); a pesar de ello, son valores cercanos, a diferencia del segundo argumento cuyo promedio de conocimiento previo fue de 2,09, y los de su elaboración en el esquema y en el resumen fueron de 1,02 y 1,43. Mientras que en el esquema sigue existiendo una débil correlación 
$(r=0,2289)$ entre el conocimiento y la adecuación y la precisión del argumento 2, en el resumen, esta se hace prácticamente nula $(\mathrm{r}=0,0661)$.

Un 20,45\% de los sujetos consideró la tesis como segundo argumento, lo que indica que si bien fueron capaces de deslindarla (cognitivamente) al generalizar la idea, no fueron capaces (metalingüísticamente) de reconocerla como tal (planteamiento central). Este dato es ilustrativo, muy probablemente, de la incidencia de la educación formal de estos estudiantes sobre la elaboración de la representación (mental), en cuanto a la organización estructural, del texto argumentativo. Fueron capaces de inferirla, pero no de determinar la premisa textual de la que partieron para hacerlo: las futuras consecuencias del cambio climático detalladas en el informe de la ONU ("en el plazo de unos pocos años, podría haber enfermedades, inundaciones, sequías, ruina agraria, éxodo humano en busca de alimentos y agua"). Como ya hemos dicho, algunos (38,63\%) asumieron una intención informativa del texto, lo que los hizo enunciar la tesis en términos igualmente informativos. De manera que si la intención del autor era presentar las diferentes versiones del fin del mundo, el segundo argumento necesariamente debía corresponderse con la tercera versión, la científica: el cambio climático como causa de destrucción. Sin embargo, y en correspondencia con ese conocimiento intuitivo de la estructura organizativa del texto, un sujeto (E32) incorporó tal premisa específica en su esquema y dos (E32 y E33), en sus resúmenes, lo que evidencia su tránsito por un proceso de redescripción representacional en el que el conocimiento en la mente estaba a un paso de hacerse conocimiento para la mente (KarmiloffSmith, 1994):

(E32) (Resumen) Distintas visiones nos aseguran un próximo fin del mundo desde las versiones arcaicas de un Apocalipsis religioso, las increíbles noticias de la ciencia ficción, hasta nuestra misma autodestrucción [...]. Así mismo los cambios climáticos sin duda alguna están contribuyendo con ese concepto del fin del mundo. Estos cambios climáticos traen consecuencias muy palpables y existentes en nuestra vida diaria: sed, hambre, sequía y una cantidad de efectos negativos para todos los seres vivos. Estos cambios afectan a algunos países más que a otros dependiendo de su posición geográfica.

Un fenómeno interesante en relación con la tesis y este segundo argumento, fue la selección, en el esquema, de una idea retóricamente llamativa pero no central, lo que dificultó su posterior articulación en el resumen: "No hay peores Apocalipsis que los de la realidad ni profecías más terribles que las del sentido común". Un 38,63 \% de los individuos la colocó en sus esquemas de manera literal o parafrástica, pero tan solo una tercera parte de ellos la insertó en sus resúmenes y, en su mayoría, lo hizo a modo de paráfrasis parciales.

En el tercer argumento termina por corroborarse la débil correlación entre conocimiento previo e identificación de los argumentos de un texto (tabla 4): $r=0,2198$ (esquema) y $r=0,1879$ (resumen). Habiendo alcanzado este argumento el más alto promedio de conocimiento $(\overline{\mathrm{x}}=2,14)$, solo fue extraído por el 59,09\% de los estudiantes. De ahí que ostente también el menor promedio de propiedad en su elaboración ( $\overline{\mathrm{x}}=0,64$, en el esquema; $\overline{\mathrm{x}}=0,77$, en el resumen). A esto puede haber contribuido, en primer lugar, y en congruencia con lo que hemos venido planteando sobre la organización conceptual desde la que algunos procesaron el texto, el que esta idea no ofrezca una nueva versión del fin del mundo; antes bien, puede habérsele considerado una información dependiente del argumento $2 \mathrm{y}$, por tanto, de la versión futura, pues casi la mitad (47,36\%) de los que no incluyeron el argumento en ambos formatos indican como planteamiento central las diferentes visiones sobre el fin del mundo.

En segundo lugar, es un argumento escasamente justificado por el autor quien lo presenta como una verdad incuestionable, sin embargo, esta estrategia pudo haber hecho dudar a algunos lectores (que podían no compartir el modelo mental del autor) de su importancia, sobre todo si poseían creencias diferentes, y hasta opuestas a las suyas (aspecto que pudo haber incidido también en la conclusión). De hecho, un $31,25 \%$ de los que incluyeron el argumento 3 en el esquema, un $27,27 \%$ de los que lo incluyeron en el 
resumen y un 10,52\% de los que no lo incorporaron en ninguno de los dos formatos creen, según el test de conocimiento previo y a diferencia del autor, que se están tomando medidas para combatir los efectos del cambio climático, específicamente en los aspectos relativos a las causas que valoraron como de mayor importancia, por ejemplo, la acumulación de gases en la atmósfera -capa de ozono- y la destrucción de la naturaleza (vegetación, aguas, etc.); razones todas que forman parte de un conocimiento cultural alcanzado principalmente a través de la educación formal y, específicamente, a través de una observación no directa de los hechos (textos escritos y audiovisuales). A su vez, Muñoz Molina se refiere a causas esencialmente referidas a la destrucción de la naturaleza (la creación de fronteras, la especulación inmobiliaria y el despilfarro del agua), que retratan una ideología ecologista que se sustenta en un conocimiento sociocultural adquirido de los medios de comunicación, de la lectura y, muy probable y fuertemente, de la observación directa de los hechos. De manera que unos - para la creación del texto- y otros - para su comprensión- han partido de sus modelos de acontecimiento y de experiencia, de participación personal (van Dijk, 1999), de los modos en que han construido el conocimiento, $y$ ello debe de haber incidido sobre la activación de tal conocimiento en ambos procesos.

Por último, pudo haberse producido una (con) fusión entre argumento y conclusión. Como dos ideas (diferentes) fueron registradas por algunos sujetos $(15,9 \%)$ y, por tanto, las escribieron en el esquema y en el resumen, otros optaron por incorporar únicamente una de ellas en sus resúmenes, ya fuera el argumento $3(8,18 \%)$ o la conclusión (25\%). En este proceso en el que la conclusión fue privilegiada, pudo tener una importante incidencia la fuerza retórica del enunciado, por lo que una gran parte de ellos tendió a copiarlo textualmente de manera parcial o total: "Lo más triste no es pensar en las cosas que ya no tienen remedio; es saber que la insensatez humana no va a dejarnos hacer las que todavía están en nuestra mano para aliviar el desastre".
Un 34,1\% de los un individuos no colocó conclusión alguna en el resumen, y un 18,18\% la re-creó (y hasta la tergiversó) o incorporó una propia, que incluso pudo ser contraria a la del autor. Partieron de un sistema de creencias (opiniones) que no lograron diferenciar de las del autor por más alejadas que estuviesen de ellas. Así, entre otros, E2 trasladó el foco del enunciado de lo que no podrá ser solucionado a lo que no tiene remedio; E8, a diferencia de Muñoz Molina, aseguró que el ser humano actuará, pero no lo hará "sino hasta que sea demasiado tarde"; E37 percibió el fin del mundo como una "sensación", y E34 vio posible su salvación. Incluso, en algunas de estas conclusiones hicieron uso de una retórica apelativa, a través de la que se dirigieron a un hipotético auditorio al que intentaron persuadir de la necesidad de generar acciones de conservación del planeta, si bien esa no es una de las estrategias utilizadas por Muñoz Molina (al menos no explícitamente), quien culmina de manera fatalista.

\section{Conclusión}

Los resultados de esta investigación indican que no hubo correlación entre el conocimiento previo comunicado por el sujeto, relativo a la temática del texto argumentativo objeto de lectura y su comprensión, en tanto identificación (ya sea por selección, generalización o construcción) de las ideas relevantes (tesis, argumentos y conclusión). Esto no significa que el estudiante no necesitara un conocimiento cultural para comprenderlo, sino que el tenerlo no fue condición suficiente para ello o, al menos, no lo fue para elaborar su macroestructura; un alto nivel de conocimiento sobre un tema no los llevó, indefectiblemente, a identificar, jerarquizar $\mathrm{y}$ articular de manera adecuada las ideas, porque además del modelo de acontecimiento, entraron en juego otros factores, como la habilidad lectoescritora y el modelo de contexto en el que insertaron el hecho comunicativo. De ahí que los argumentos que resultaron con el mayor promedio de conocimiento fueran, a su vez, los de menor nivel de identificación adecuada y precisa; o que el nivel global de conocimiento previo fuera superior al de 
la competencia general para resumir el texto que, por lo demás, fue baja.

La experiencia previa de acercamiento a textos expositivos y la finalidad del proceso de resumir fueron agentes de gran incidencia sobre la tarea realizada, lo que está en relación con el modelo de contexto desde el que gran parte de los estudiantes se aproximó a la situación de lectura. Según el modelo contextual que suelen tener de prueba de diagnóstico, el profesor evalúa la posesión de un conocimiento especializado, básicamente declarativo. Así, teniendo el aprendiz un conocimiento temático, representado a través de su modelo de acontecimiento, que pudo o no haber sido explicitado con anterioridad, tal conocimiento no necesariamente se activó -y si lo hizo pudo no haberlo hecho de manera adecuada-cuando fue requerido para procesar el conjunto de ideas y derivar una estructura semántica jerarquizada en función de su relevancia. El procedimiento utilizado en un número considerable de casos fue el de acercamiento al texto expositivo $y$, en concreto, al del conocimiento especializado; asumieron tanto su rol (participante) de lectores, como su rol (profesional) de estudiantes.

En consecuencia, algo menos de una tercera parte de los sujetos que elaboraron la tesis (aunque ciertamente en todos hubo un bajo promedio de nivel de adecuación y precisión) la entendió como argumento. El resto, al percibir una intención informativa, procedió a estructurar la idea en términos de tema. Asimismo, aquellos que atribuyeron la función de tesis al primer argumento, en cuanto a las visiones de la religión y de la ciencia ficción sobre el fin del mundo, o que construyeron una estructura mayor que incluyera también la visión científica, optaron por una superestructura (expositiva) consistente en una generalización temática (versiones sobre el fin del mundo) explicada a través de una secuencia cronológica de hechos (pasado, presente y futuro), en lugar de la comparativa utilizada por el autor, y a partir de la cual la referencia de unos hechos del pasado (fin del mundo: Apocalipsis y ciencia ficción) permitía establecer comparaciones con hechos del presente (cambio climático) que, a su vez, podrían dar origen a otros hechos en el futuro (fin del mundo a causa del cambio climático), y por tanto, inferir la tesis. Ciertamente, comprender el texto requirió comprenderlo como la manifestación de una opinión (van Dijk, 1999). Por último, el identificar la idea relativa a la tesis no necesariamente los llevó a seleccionar el segundo o tercer argumento, así como a determinar con precisión la conclusión, en congruencia con la posición crítica esgrimida por el autor.

Los datos muestran, además, una correlación más alta entre los niveles de conocimiento previo y de adecuación y precisión de tres de las cinco macroproposiciones (tesis y argumentos 2 y 3 ) del esquema (previo) que del resumen. ¿Se debió, acaso, tan solo a la (falta de) habilidad de escritura, necesaria para articular las ideas? ¿Por qué se incluyeron en algunos resúmenes ideas no presentes en los esquemas o, por el contrario, se excluyeron las que antes habían sido consideradas?; de no ser elididas, ¿por qué pudieron perder la precisión con que habían sido construidas con anterioridad? Debido a que el conocimiento es una creencia representada en la memoria de largo plazo "usada parcialmente y aplicada" por la de corto plazo (van Dijk, 2005), otro de los aspectos cruciales de este proceso de lectoescritura parecería no estar tanto en el grado de conocimiento que se posee, como en el que efectivamente se activa en cada uno de sus momentos en función del modelo de contexto: durante la lectura, para la identificación de las ideas, y a lo largo del proceso de escritura, con la finalidad de determinar su presencia/ausencia en los nuevos ciclos de procesamiento durante la planificación del resumen (entendiendo el esquema como tal) y en su escritura efectiva. Entonces, ¿ se mantiene activo redimensionándose en virtud de nuevos factores?; ¿cuáles, específicamente? ¿Se desactiva?; ¿qué incide en ello? ¿Se activa otro conocimiento?; ¿a qué causas obedece?

Probablemente algunos de esos factores estén relacionados con los criterios a través de los cuales el conocimiento es adquirido. Los resultados relativos al texto en general y, específicamente, al tercero de los argumentos nos lleva a plantearnos cuánto y cómo inciden los conocimientos adquiridos a través de criterios epistemológicos y de 
sentido común -observación directa, fuentes fiables e inferencias- (van Dijk, 2005), en el proceso de comprensión lectora, específicamente cuando se trata de determinar la opinión del otro. Por consiguiente, habría que tener en cuenta, además del conocimiento que se posee previamente y que se ha construido fundamentado en tales criterios, otros como el que en efecto se activa porque ya se tiene y el que se adquiere a partir de la lectura del propio texto, proceso este último en el que las inferencias han de tener un papel fundamental.

Finalmente, hemos de advertir que los resultados relativos a la selección de la conclusión parecerían indicar que las creencias, en términos de opinión, podrían influir sobre la comprensión del texto argumentativo. Habría que determinar, por tanto, cómo y en qué medida lo hacen.

\section{Bibliografía}

Alonso Tapias, J. (2005). Claves para la enseñanza de la comprensión lectora, en Revista de educación, núm. extraordinario, 63-93.

Bernardo, A. B., Stenberg, R. J. (1991). Contextual reference domains and verification: What is relevant and what is not in sentence verification, en Journal of Memory and Language, 30, 664-684.

Castejón, J. L., Prieto, M. D., Pérez, A. M., Gilar, R. (2004). El rol del conocimiento y de las habilidades intelectuales generales en la adquisición del aprendizaje complejo, en Psicothema, 16(4), 600-605. Disponible en: http://www.psicothema.com/pdf/3038.pdf.

González Nieto, L. (2001). Teoría lingüística y enseñanza de la lengua. (Lingüística para profesores). Madrid: Cátedra.

Gray, S., Rinck, M., McNamara, T., Bower, G., Morrow, D. (1993). Mental models and narrative comprehension: Some Quaifications, en Journal of Memory and Language, 32, 141-154.

Karmiloff-Smith, A. (1994). Más allá de la modularidad. Madrid: Alianza Editorial.

Kahmi-Stein, L. (1997). Las estrategias de resumen de alumnos de "alto riesgo", en Lectura y vida, 4, 17-24.

Kintsch, W., van Dijk, T. (1978). Toward a Model of Text Comprehension and Production, en Psychological Review, 85, 364-394.
Kletzien, S. (1991). Strategies used by good and poor comprehenders reading expository text of differing levels, en Reading Research Quarterly, XXVI, 67-86.

Long, D. L., Oppy, B. J., Seely, M. R. (1994). Individual Differences in the Time Course of Inferential Processing, en Journal of Experimental Psychology: Learning, Memory and Cognition, 20, 1456-1470.

McCormick, S. (1992). Disabled Readers' Erroneous Responses to Inferential Comprehension Questions: Description and Analysis, en Reading Research Quarterly, 27, 55-77.

McGinitie, W., Maria, K., Kimmel, S. (1986). El papel de las estrategias cognitivas no-acomodaticias en ciertas dificultades de la comprensión de lectura. En E. Ferreiro y M. Gómez (comp.). Nuevas perspectivas sobre los procesos de lectura y escritura, $4^{\mathrm{a}}$ ed. (pp. 29-49). México: Siglo XXI.

McNamara, D. (2004). Aprender del texto: efectos de la estructura textual y las estrategias del lector, en Revista Signos, 37(55), 19-30. Disponible en: http://www.scielo.cl/scielo.php?pid=S071809342004005500002\&script=sci_arttext.

Núñez, R., Del Teso, E. (1996). Semántica y pragmática del texto común. Producción y comentario de textos. Madrid: Cátedra.

Pozo, J. I. (2002). La adquisición de conocimiento científico como un proceso de cambio representacional, en Investigações em ensino de ciências, 7 (3), 245-270. Disponible en: http://www.if.ufrgs.br/public/ensino/ vol7/n3/v7_n3_a5.htm.

Sánchez Miguel, E. (1993). Los textos expositivos. Estrategias para mejorar su comprensión. Madrid: Santillana, Aula XXI.

Solaz-Portolés, J. J., Sanjosé López, V. (2007). Resolución de problemas, modelos mentales e instrucción $R e$ vista electrónica de enseñanza de las ciencias, 6(1), 70-89. Recuperado de: http://saum.uvigo.es/reec.

Solaz-Portolés, J. J. y Sanjosé L., V. (2008). Conocimientos y procesos cognitivos en la resolución de problemas de ciencias: consecuencias para la enseñanza. Magis. Revista Internacional de Investigación en Educación, 1, 147-162. Recuperado de: http://www.javeriana. edu.co/magis/numero-uno/magis-3-arti-1-8-resumen-solaz-portoles.html.

Solé, I. (1992/2006). Estrategias de lectura. $16^{\circ}$ ed. Barcelona: GRAÓ, ICE de la Universidad de Barcelona.

Van Dijk, T. (1999). Ideología. Barcelona: Gedisa. 
Van Dijk, T. (2000). El estudio del discurso. En: T. van Dijk (comp.). El discurso como estructura y proceso (pp. 21-65). Barcelona: Gedisa.

Van Dijk, T. (2002). Tipos de conocimiento en el procesamiento del discurso. En Parodi, G. (ed.). Lingüística e interdisciplinariedad: desafíos del nuevo milenio. Ensayos en honor a Marianne Peronard (pp. 43-66). Valparaíso: Ediciones Universitarias.

Van Dijk, T. (2005). Discurso, conocimiento e ideología. Reformulación de viejas cuestiones y propuesta de algunas soluciones nuevas. Cuadernos de información y comunicación, 10, 285-318. Recuperado de: http://revistas.ucm.es/index.php/CIYC/article/view/ CIYC0505110285A/7303

Van Dijk, T. (2010). Discurso, conocimiento, poder y política. Hacia un análisis crítico epistémico del discurso. Revista de investigación lingüística, 13, 167215. Recuperado de: http://revistas.um.es/ril/article/ view/114181/108121

Wine, P.; Grahm, L. y Prock, L. (1993). A model of poor readers' text-based inferencing: Effects of explanatory feedback. Reading Research Quarterly, 28, 53-65.

Zipitria, I.; Elorriaga, J.A. y Arruarte, A. (2007). Hacia la automatización de la evaluación de resúmenes desde la experiencia cognitiva. Revista Iberoamericana de Informática Educativa, 5, 49-61. Recuperado de: http://161.67.140.29/iecom/index.php/IECom/ article/viewFile/16/10. 